\title{
El nuevo modelo económico en México 2018
}

\section{The new economic model in Mexico 2018}

DOI: 10.46932/sfjdv3n1-057

Received in: Jan 30st, 2021

Accepted in: Feb 1th, 2022

\section{Saúl Robles Soto}

Doctor en Ciencias Económicas, Docente-Investigador de la Unidad Académica de Economía de la Universidad Autónoma de Zacatecas, México.

E-mail: saul.robles@ymail.com

\section{Rigoberto Jiménez Díaz}

Estudios del Desarrollo, Docente-Investigador de la Unidad Académica de Economía de la Universidad Autónoma de Zacatecas, México.

E-mail: rigalfa@yahoo.com

\section{Claudia Esther del Rosario Aguilar Torres}

Doctora en Ciencia Política, Docente-Investigador de la Unidad Académica de Economía de la Universidad Autónoma de Zacatecas, México.

E-mail: clauaguila@yahoo.com

\section{Luis Humberto Ortiz Romero}

maestro en informática administrativa, Docente-Investigador de la Unidad Académica de Economía de la Universidad Autónoma de Zacatecas, México

\section{RESUMEN}

En México, a partir del primero de diciembre del año 2018, se pretende implementar un nuevo modelo económico que cambie al impuesto en los últimos 36 años. El trabajo que se presenta es una investigación desarrollada por el Cuerpo Académico de Teoría Económica de la UAEUAZ ${ }^{1}$, tiene como objetivos señalar de manera puntual los logros y fracasos del modelo neoliberal en México, poniendo énfasis en los principales resultados que generó entre otros mayor pobreza, inversión pública insuficiente, deuda pública alarmante y un desempleo muy notorio. Se presentan una serie de variables macroeconómicas que dan sustento a la hipótesis central de un modelo económico fracasado que es necesario revertir en el mediano plazo. Las conclusiones serán importantes para tratar de implementar un cambio importante en el papel del estado en los ejes fundamentales de la economía mexicana como factor fundamental que devuelva los índices de crecimiento y bienestar que estuvieron presentes tiempo atrás.

Palabras clave: México, pobreza, inversión, modelo, economía.

\begin{abstract}
In Mexico, as of December 1, 2018, it is intended to implement a new economic model that changes the one imposed in the last 36 years. The work presented is a research developed by the Academic Body of Economic Theory of the UAEUAZ, has as objectives to point out in a timely manner the achievements and failures of the neoliberal model in Mexico, emphasizing the main results that generated among others
\end{abstract}

\footnotetext{
${ }^{1}$ Unidad Académica de Economía de la Universidad Autónoma de Zacatecas, México
} 
greater poverty, insufficient public investment, alarming public debt and a very notorious unemployment. A series of macroeconomic variables are presented that support the central hypothesis of a failed economic model that must be reversed in the medium term. The conclusions will be important to try to implement an important change in the role of the state in the fundamental axes of the Mexican economy as a fundamental factor that will bring back the growth and welfare indexes that were present in the past.

Keywords: Mexico, poverty, investment, model, economy.

\section{INTRODUCCIÓN}

Para hablar de problemas económicos en México durante los últimos años, debemos hacer mención a Capdeville y Dutrénit (2012), el cual manifiesta que la crisis económica internacional ha planteado la necesidad de reconsiderar el papel del estado en el desarrollo económico. La desregulación de la economía mundial, en particular en relación con el sector financiero, ha generado una elevada inestabilidad y ha conducido a una crisis con efectos adversos significativos sobre la economía mexicana. Sin embargo, el problema fundamental de la economía en México no es la crisis económica internacional, sino la situación de estancamiento y deterioro en el bienestar social como consecuencia de tres décadas de mal desempeño económico, regulación de la economía que limitó la promoción del desarrollo productivo por parte del estado. ${ }^{2}$

En base a lo anterior, el nuevo gobierno encabezado por Andrés Manuel López Obrador (AMLO), a partir del primero de diciembre del año 2018, pretende en lo inmediato modificar al mal desempeño de la economía nacional y obliga a repensar y reevaluar la función del estado en la economía.

Derivado de lo anterior, presentamos cuatro variables fundamentales del modelo económico neoliberal implementado en México desde la década de los años 80’ que han resultado muy perjudiciales para la mayoría de la población, éstas son pobreza, inversión pública, deuda pública y desempleo.

La finalidad del trabajo desarrollado por académicos de la UAEUAZ, permitirá aportar elementos importantes en términos económicos que desde la oficialidad nos presentan continuamente en diversos espacios, que al compararlos con los manejados por la academia nos permiten comprobar lo erróneo del modelo neoliberal implementado en los últimos seis sexenios.

Debemos tener en cuenta que esta investigación es resultado de la revisión periódica de información oportuna aparecida en organismos especializados en la economía mexicana, textos, revistas, tesis de diversos niveles y periódicos con información económica.

\footnotetext{
${ }^{2}$ Capdeville M y Dutrénit G, Políticas para el desarrollo productivo y la innovación: desafío y oportunidad para la economía mexicana, p. 153, capítulo del texto Nueva estrategia de industrialización, Volumen 7, coordinado por José Luis Calva, Ed. JP, 2012. México.
} 
Dichas investigaciones desde el año 2009 la hemos realizado de manera permanente, dando a conocer los resultados obtenidos en congresos, foros y coloquios.

En base a lo anterior, presentamos a continuación las cuatro variables señaladas para el modelo económico mexicano.

\section{POBREZA}

En datos proporcionados por el gobierno federal en el mes de marzo del año 2019, se manifiesta abiertamente que existen varios Méxicos por la desigualdad que se vive en el país, por lo que se requiere con urgencia un viraje económico.

Durante los años que comprenden del 2008 al 2018, la pobreza en México aumentó en 4.2 millones de personas, al pasar de 49.5 millones a 53.7 millones. ${ }^{3}$

Estas cifras las podemos observar en la gráfica número 1, referida a la pobreza en México durante el período 2008-2018 en millones de personas.

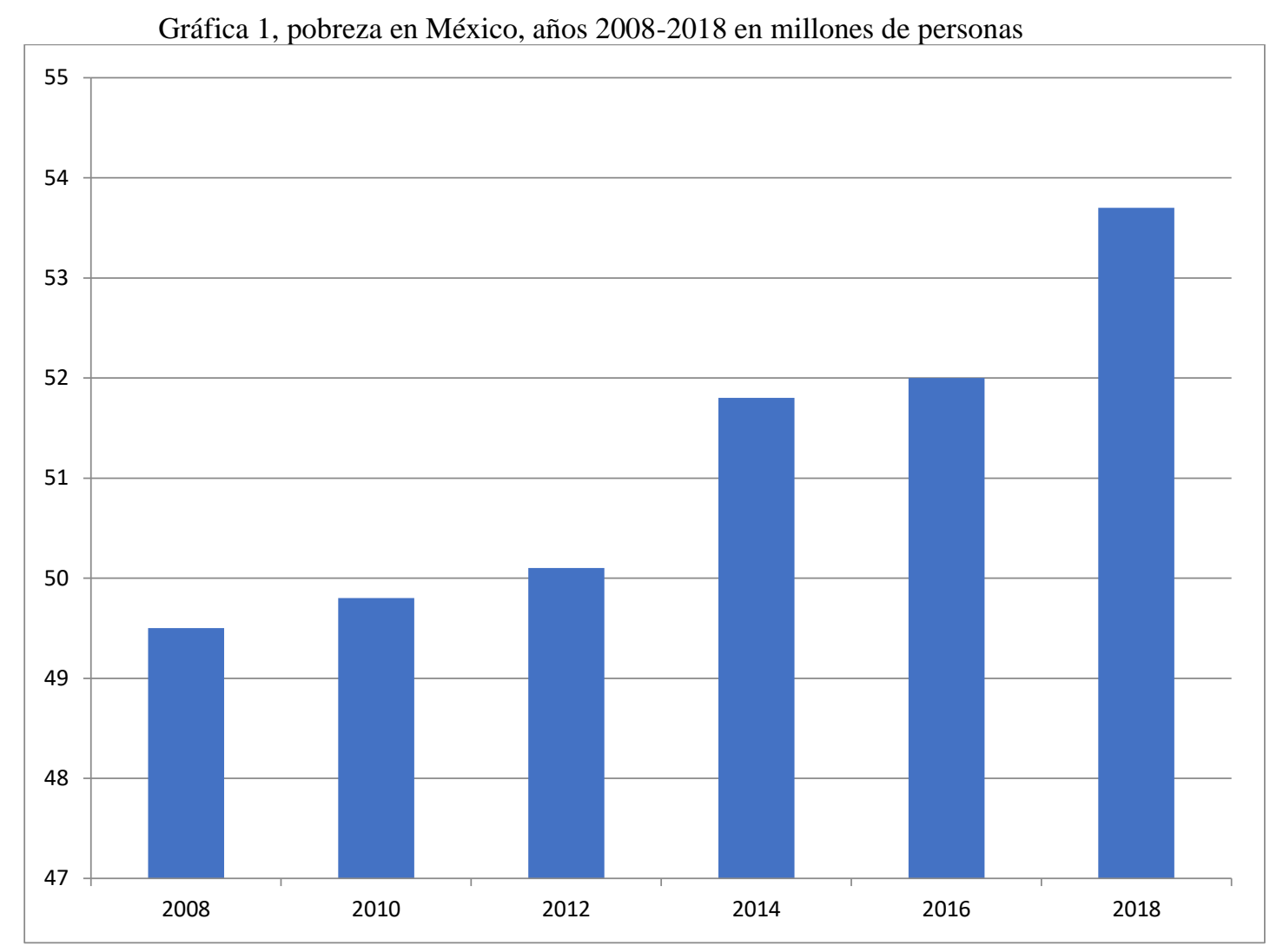

Fuente: Elaboración propia con datos de: LEA, INEGI, SE, varios años, 2019

Como es natural, en 13 años, en lugar de disminuir en términos cuantitativos la pobreza en México, se hace cada vez más grande sin visos de haberse disminuido.

\footnotetext{
${ }^{3}$ Consejo nacional de Evaluación de la Política de Desarrollo Social, 17 de marzo del año 2019.
} 
En la medida que no disminuye la pobreza en México, ahora la pregunta sería y cómo se combate de manera frontal para disminuirla tal como se refleja en la gráfica número 1.

Si se tienen los índices de pobreza señalados, no solamente se tiene pobreza, sino lo más grave, también la desigualdad.

Los últimos datos al respecto señalados por el gobierno que encabeza el presidente Andrés Manuel López Obrador, (AMLO), el 30 por ciento de la población más rica del país se queda con más de 60 por ciento del ingreso nacional, por lo que la sociedad mexicana es una de las más desiguales en el mundo, entonces, la desigualdad implica injusticia muy marcada en México.

Datos representativos de la desigualdad que originó más pobreza en al país mexicano los tenemos a continuación:

En México, alrededor de 8 mil 895 mexicanos lograron ingresar durante el gobierno de Enrique Peña Nieto (EPN) en el segmento de millonarios con fortunas superiores a los 500 millones de pesos, revelan cifras oficiales de inversionistas que operan mediante casas de bolsa.

Al cierre del sexenio de EPN, eran un total de 24 mil 181 mexicanos con registros de fortunas de 500 millones de pesos, o más, cuando en 2013, al inicio del gobierno, el mismo grupo lo conformaban 14 mil 486 millonarios, es decir, un crecimiento de 9 mil 695 más en solamente seis años, lo que se traduce en 1 mil 615 nuevos ricos mexicanos cada año en el gobierno de EPN. ${ }^{4}$

El selecto grupo de millonarios en México tiene registradas oficialmente cuentas de orden que involucran recursos por 9 billones 981 mil 426 millones de pesos, cifra que representa alrededor de 59 por ciento del producto interno bruto (PIB) del país, es decir, la riqueza que se genera en un año en todas las actividades económicas en México.

De acuerdo con los registros oficiales, la riqueza se encuentra concentrada, en 147 mil 878 personas físicas y 39 mil 68 personas morales con fortunas hasta por 15 millones de pesos; con entre 15 y 50 millones se tienen registros de 5 mil 508 personas físicas y mil 907 morales; con más de 50 millones y hasta 100 millones de pesos hay 2 mil 472 personas físicas y 833 morales; con más de 100 millones y hasta 250 millones de pesos se tiene registro de 3 mil 729 personas físicas y mil 389 morales; con 250 millones y hasta 500 millones de pesos la información oficial da cuenta del registro de 4 mil 825 personas físicas y 822 morales, mientras quienes tienen más de 500 millones de pesos totalizan 20 mil 579 personas físicas y 4 mil 854 morales.

También se tiene identificados por nacionalidad a los inversionistas que operan en el mercado nacional en casas de bolsa para gestionar la adquisición, venta o tenencia de acciones de empresas cotizadas en la Bolsa Mexicana de Valores: 172 mil 179 inversionistas son mexicanos; 2 mil 285

\footnotetext{
${ }^{4}$ Período 2012-2018
} 
estadunidenses, 195 canadienses y 2 mil 997 de otras nacionalidades con fortunas de hasta 15 millones de pesos.

En el siguiente estrato, con más de 15 millones y hasta 50 millones de pesos, 6 mil 951 son mexicanos, 60 estadunidenses y 383 de otras nacionalidades; el segmento con más de 50 millones y hasta 100 millones está compuesto por 4 mil 851 mexicanos, 50 estadunidenses y 383 de otras nacionalidades; con más de 100 millones y hasta 250 millones de pesos, hay 5 mil 508 mexicanos, 41 de origen estadunidense, seis canadienses y 337 de otras nacionalidades; quienes tienen más de 250 millones y hasta 500 millones de pesos suman 5 mil 348 mexicanos, 64 de nacionalidad estadunidense, cinco canadienses y 978 de otras, mientras que los que se encuentran en la cima, con fortunas superiores a 500 millones de pesos, son 24 mil 381 mexicanos, 457 estadunidense, 26 canadienses y mil 982 de otras nacionalidades.

Gráfica número 2, evolución de la pobreza en materia de ingresos, período 1992-2016 en porcentaje

\section{Evolución de la población en pobreza en materia de ingresos 1992-2016 Porcentaje}

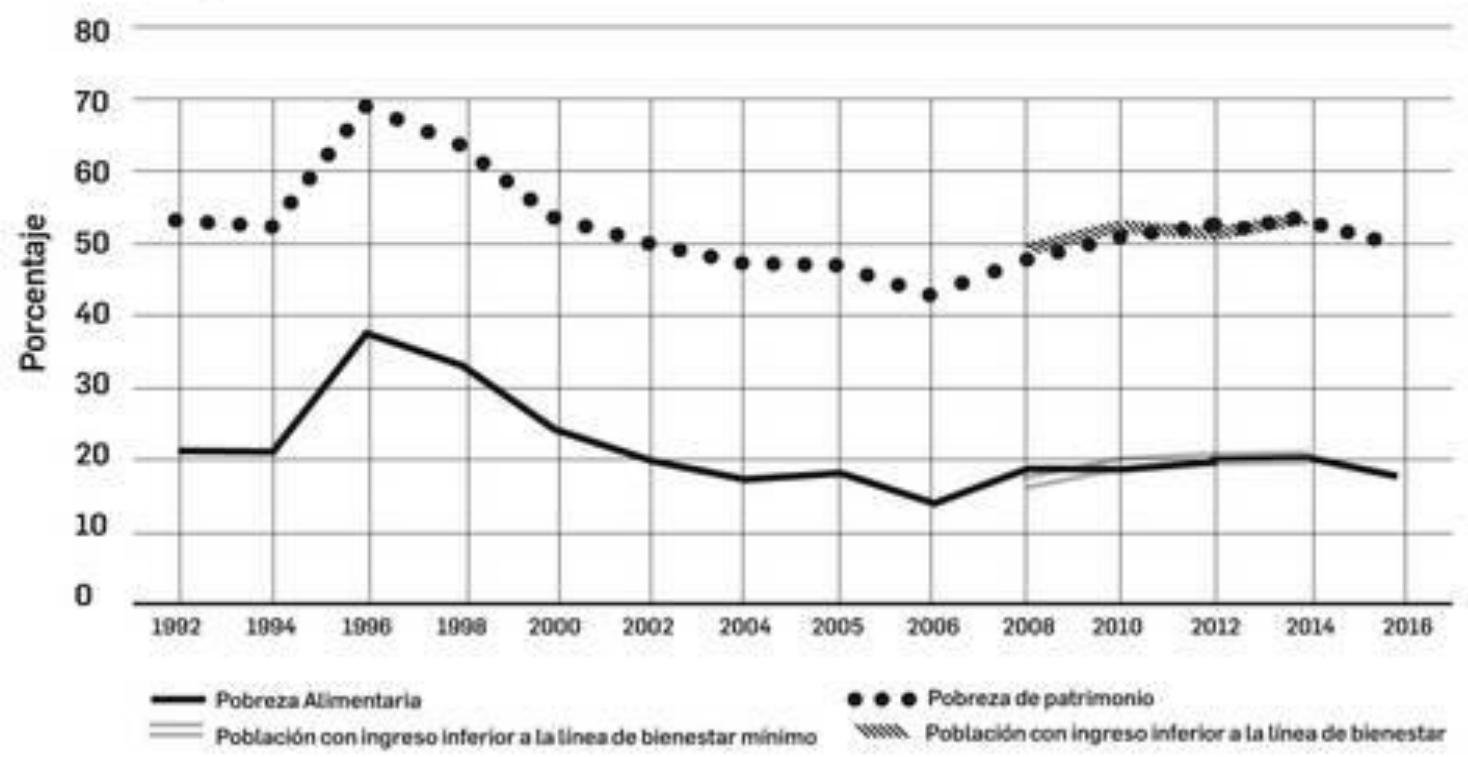

Fuente: Periódico la Jornada, Angélica Enciso L., lunes 9 de julio de 2018, p. 11.

La gráfica número 2, refleja que 6 mil 489 programas sociales de los tres niveles de gobierno, el porcentaje de la población con bajos ingresos es prácticamente el mismo de hace 26 años.

Desde el año de 1992, 53.1 millones de mexicanos carecían de recursos para alcanzar a cubrir sus necesidades básicas, esta cantidad de personas señaladas aumentó para el año 2016 hasta 62 millones.

Con el nuevo gobierno federal, se pretende que los programas para abatir la pobreza no han funcionado porque han sido diseñados e implementados desde una visión paternalista y operados mediante mecanismos clientelares. 


\section{EMPLEOS}

Una característica importante del gobierno federal bajo el sexenio de EPN lo constituye lo referente al empleo en México, se presumió en varios eventos al final de su mandato que ha sido el presidente que generó lo mayor cantidad de empleos en los últimos años.

En los datos de la gráfica número 3, se presentan los años del 2012 al 2018, en los cuales se especifica claramente que el único año en el cual disminuyó el nivel de empleo fue el año 2015 con 815. 9 mil empleos, cifra inferior al alcanzado en el año 2014 con 827.2 mil nuevos empleos.

Gráfica 3, Empleos generados en México, 2012-2018 en miles

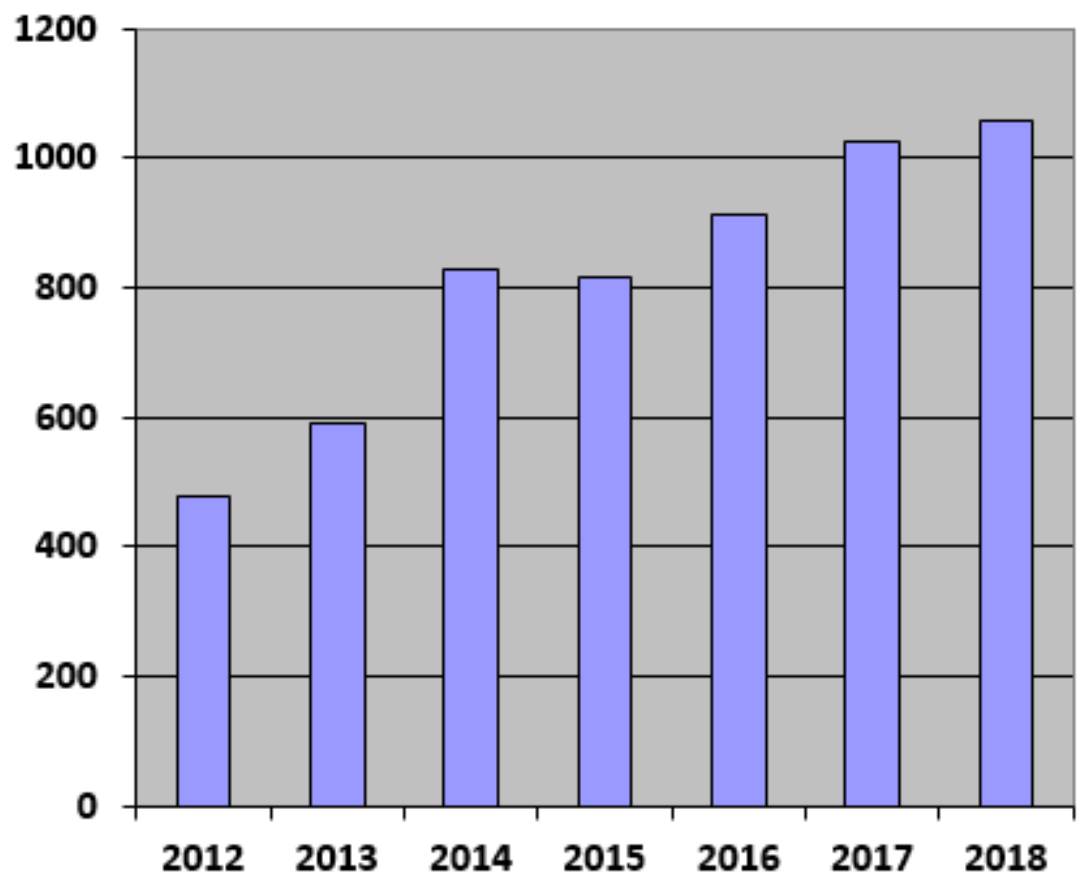

Fuente: elaboración propia con datos del INEGI, LEA, STPS, SE, varios años, 2019.

Es necesario tener en cuenta que el modelo neoliberal implementado en México desde la década de los años 80's vaticinaba que se generarían en promedio un millón de empleos cada año, por lo que las cifras presentadas en la gráfica para el período 2012-2018 no fueron del todo cumplidas, excepto para los años 2017 y 2018.

\section{IVERSIÓN PÚBLICA}

En los gobiernos de corte neoliberal, se contuvo la inversión pública, con el consiguiente adelgazamiento del aparato gubernamental que fue suplido por obras concesionadas a la iniciativa privada, ello aumentó los beneficios para un segmento poblacional muy escaso.

El desprecio hacia la inversión pública en el gobierno de EPN se anota en la gráfica número 4, donde se presenta una continua disminución en sus 6 años de gobierno. 
Ello ocasionó un abandono gradual en sectores económicos claves para el buen funcionamiento de la economía en México al no considerar al papel de inversionista el sector público el cual debe ser fundamental para incentivar las actividades productivas en el país. Al no ser considerado un factor clave la inversión pública para el sexenio del presidente EPN durante el período 2012-2018, se obtuvieron resultados muy precarios en términos de crecimiento y desarrollo económicos.

Debemos tener en cuenta que la apreciación referida a disminuir constantemente la inversión pública en México por parte del modelo económico neoliberal obedece también a los lineamientos establecidos por diversos organismos internacionales que argumentan la necesidad de adelgazar lo más posible la participación del estado en las actividades económicas.

Gráfica número 4, Inversión pública en México como porcentaje del PIB, período 2012-2018.

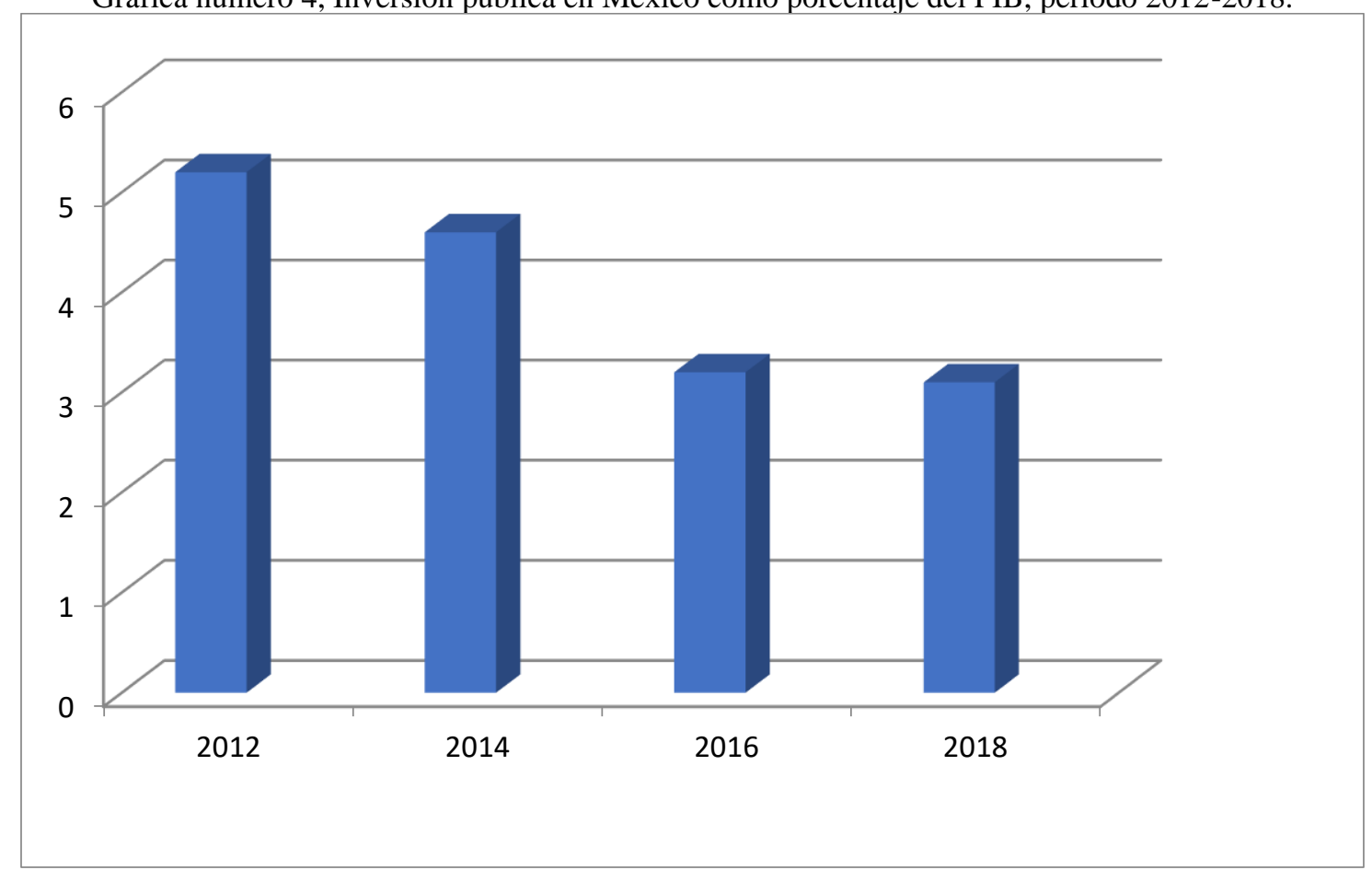

Fuente: elaboración propia con datos del INEGI, LEA, STPS, SE, varios años, 2019.

\section{DEUDA PÚBLICA}

En contraposición a la disminución de la inversión pública, se incrementa de sobremanera la deuda pública en el sexenio de EPN, al grado de considerarla la más abultada de la historia mexicana. El resultado del crecimiento de la deuda pública en México se originó por el consabido interés de gastar más de lo que se obtenía de ingresos en el país, esto es, la recaudación del gobierno federal no fue la adecuada a pesar de los continuos señalamientos del gabinete económico peñista de que se aumentó la base gravable de impuestos en los últimos seis años. 
En el mes de diciembre del año 2012, el monto de la deuda pública se ubicaba en 4.75 billones de pesos, quedando en el mes de diciembre del año 2018 (al final de la administración de EPN) en una cantidad de 10 billones de pesos, un crecimiento en seis años de 5.25 billones de pesos, esto es, un promedio anual de 875 mil millones de pesos, cifra en verdad alarmante.

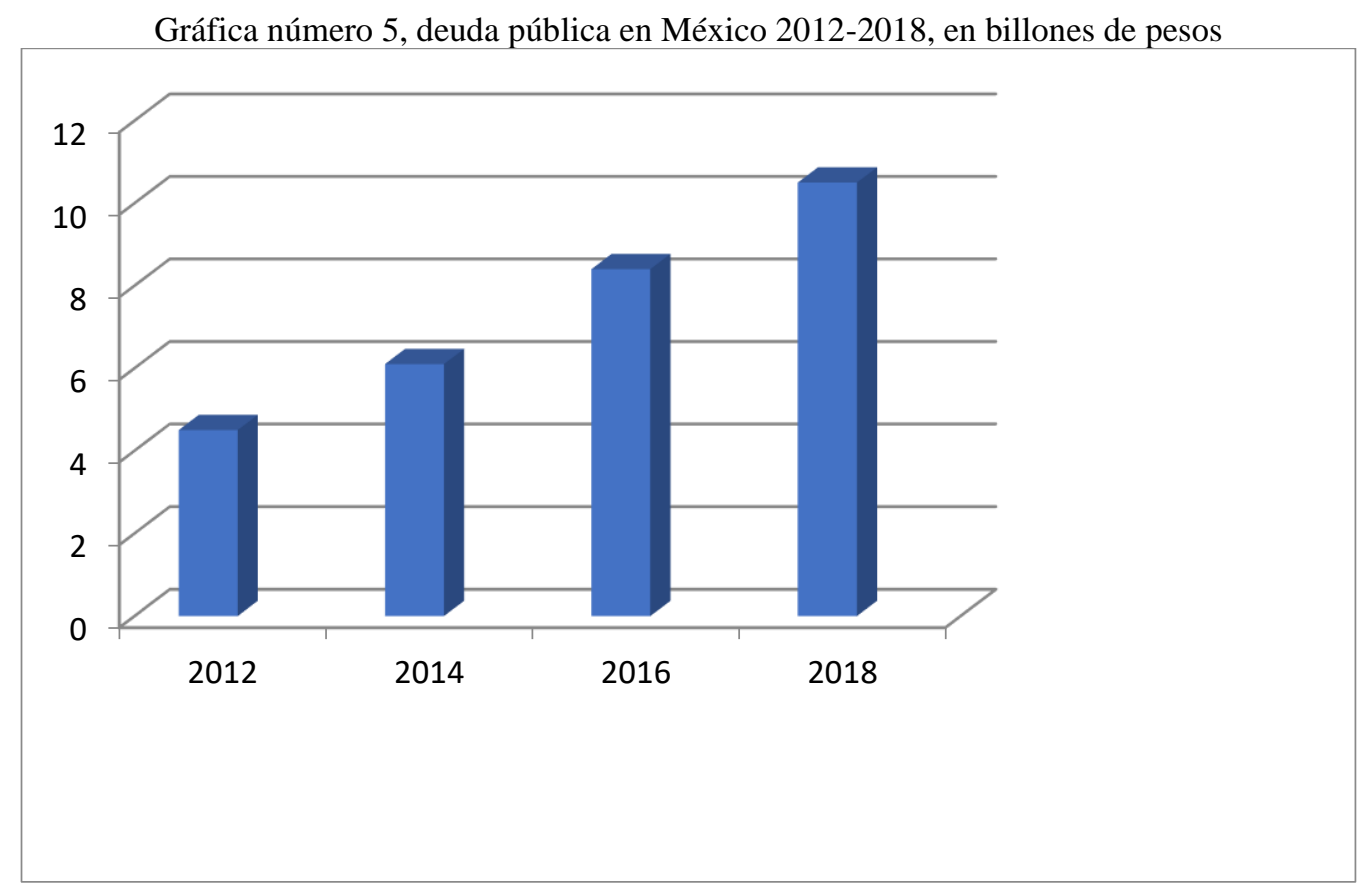

Fuente: Elaboración propia, con datos de LEA, SHCP, SE, INEGI, varios años, 2019

Es en verdad fue preocupante el aumento de la deuda pública y las consecuencias que tiene su pago y respaldo a toda costa por parte del gobierno de EPN. Pues no sólo han sido consecuencia de ello la reducción presupuestal de sectores importantes, sino que a su vez el Gobierno de Peña Nieto ha diseñado e implementado reformas de orden neoliberal que intensifican los hostigamientos a que es sometida la sociedad mexicana.

El pueblo mexicano nos ha brindado importantes lecciones de lucha en la historia latinoamericana, y es hora que como ya lo ha hecho antes se levante en contra de esa herramienta de dominación, que es la deuda pública, pues de lo contrario en unos meses/años serán las Instituciones Financieras Internacionales, una vez más, las que dictaran el proseguir del país imponiendo y profundizando las políticas de austeridad. Es que basta con recordar las palabras del Presidente Peña Nieto cuando agradecía a las misiones enviadas por diferentes países para colaborar con las labores de rescate por el terremoto de septiembre de 2017, y sostenía que su forma de agradecimiento con estos países sería con solicitudes de créditos bilaterales para responder a las exigencias de la población. Así de odioso es el instrumento de la deuda, pues en el marco del capitalismo no se puede vislumbrar una ayuda desinteresada sino que a nivel de los Estados la ayuda normalmente está condicionada, como ya se había visto en muchos otros países. 


\section{CONCLUSIONES}

Es indudable que el modelo neoliberal en México ocasionó una serie de inconvenientes para la economía mexicana que lejos de beneficiar a la población en general, originó mayor pobreza, desempleo, desigualdad, falta de inversiones públicas y un aumento considerable en la deuda pública que debemos pagar todos los mexicanos sin haber tenido los beneficios esperados.

Con la llegada del nuevo gobierno federal desde el 1 de diciembre del 2018 se tiene la esperanza de cambiar de manera radical el uso de variables económicas que permitan obtener mayor crecimiento y desarrollo en México, es la gran apuesta del presidente Andrés Manuel López Obrador el cual pretende revitalizar la función del estado para beneficio de todos los mexicanos y no solamente de un segmento muy reducido de la población. 


\section{BIBLIOGRAFÍA}

ALEgRíA, A, Periódico La Jornada, Domingo 17 de marzo de 2019, p. 18

Burnes A. y Padilla J. M., Zacatecas Hoy, nueve ensayos sobre economía y sociedad, Ed. UAZ, México, 2014.

Calva J, Nueva Estrategia de industrialización, Análisis Estratégico para el Desarrollo, volumen 7, Ed. JP y el CNU, México, 2012.

ENCISO, A, Periódico La Jornada, Lunes 9 de julio de 2018, p. 11

Instituto Nacional de Estadística Geografía e Informática, varios años, México, 2019.

Laboratorio de Economía Aplicada, Unidad Académica de economía de la Universidad Autónoma de Zacatecas, varios años, México, 2019.

Robles S., Alcances y limitaciones del neoliberalismo en México y Zacatecas, 2000-2014, Ed. UAZ, México, 2015

El fracaso del modelo neoliberal en México 2000-2016 ponencia, 4to. Congreso Latinoamericano de Ciencias Sociales, UASC, UAZ, México, junio 2016.

El salario mínimo en México, repercusiones económicas 2016, Ponencia, Congreso Academia Journals, Fresnillo, Zacatecas, México, febrero 2017.

Secretaría de Economía, varios años, México, 2019.

Secretaría de Hacienda y Crédito Público, varios años, México, 2019.

Secretaría de Trabajo y Previsión Social, varios años, México, 201. 